\title{
Wearable bioimpedance for continuous and context-aware clinical monitoring
}

\author{
Abhilash Guru Dutt, Michaela Verling and Walter Karlen, Senior Member, IEEE
}

\begin{abstract}
Bioimpedance monitoring provides a non-invasive, safe and affordable opportunity to monitor total body water for a wide range of clinical applications. However, the measurement is susceptible to variations in posture and movement. Existing devices do not account for these variations and are therefore unsuitable to perform continuous measurements to depict trend changes. We developed a wearable bioimpedance monitoring system with embedded real-time posture detection using a distributed accelerometer network. We tested the device on 14 healthy volunteers following a standardized protocol of posture change and evaluated the agreement with a commercial device. The impedance showed a high correlation $(r>0.98)$, a bias of $-4.5 \Omega$, and limits of agreement of -30 and $21 \Omega$. Context-awareness was achieved with an accuracy of $94.6 \%$ by classifying data from two accelerometers placed at the upper and lower leg. The calculated current consumption of the system was as low as $10 \mathrm{~mA}$ during continuous measurement operation, suggesting that the system can be used for continuous measurements over multiple days without charging. The proposed motion-aware design will enable the measurement of relevant bioimpedance parameters over long periods and support informed clinical decision making.
\end{abstract}

Keywords: bioimpedance, wearable devices, fluid monitoring, actimetry, context-awareness, internet of medical things

\section{INTRODUCTION}

A bioimpedance analyzer is a bioelectrical instrument to estimate body composition by measuring the electrical response of the tissues to the injection of a weak alternating current. Bioimpedance is sensitive to changes in total body water and fluid shifts, but is also used to estimate fatfree body mass. Bioimpedance has been suggested for a wide range of clinical applications ranging from impedance plethysmography [1], fluid status estimation [2], dengue management [3], up to wound healing [4]. Bioimpedance assessments are an interesting technique as it is non-invasive and lower in cost compared to many other approaches, such as esophageal doppler monitoring or dual-energy X-ray absorptiometry (DEXA).

A state-of-the-art bioimpedance setup consists of tetra polar configuration with 4 wires attached to the human body. The current is injected into the body (load) with one pair of electrodes and the other pair is used to measure the voltage across the load. The signal is dependent on anthropometric factors such as age, sex and ethnicity. Postural changes may cause small shifts of fluids across body compartments and can alter the bioimpedance reading [5].

This project was funded by the Swiss National Science Foundation (150640), ETH Global, and the Royal Academy of Engineering

Mobile Health Systems Lab, Institute of Robotics and Intelligent Systems, Department of Health Sciences and Technology, ETH Zurich, Zurich, Switzerland (email: walter.karlendieee.org)
Furthermore, bioimpedance measurements are distorted by motion artifacts [6]. Existing technologies do not account for such movements and also ignore postural variations. In addition, commercial devices are rather bulky, unreasonably expensive, and inconvenient to use, in particular for continuous measurements. However, continuous monitoring is an essential requirement in clinical settings to detect transitions of physiological states [7]. This might explain the limited uptake of bioimpedance in clinical routines.

The goal of this work was to develop and test a novel integrated bioimpedance measurement system that is wearable, low power and affordable, and that enables context-based, continuous, and real-time bioimpedance assessments.

\section{DESIGN}

We designed the device to include several functionalities to improve its possible clinical usage and overcome the limitations of existing systems that prevent continuous bioimpedance measurement. These contributions include an integrated, low-power hardware design that includes internet of medical things functionality and a sensor network of distributed accelerometers. Furthermore, the software includes sensor fusion and context awareness that enables continuous measurements independent of motion or position changes.

\section{A. Hardware}

1) CPU and User Interface: The processing module of the system was a BLE654 (Laird Connectivity) which was based on the nRF52840 chip set (Figure 1). It featured a 32 bit ARM Cortex-M4 CPU which was used to acquire data from the bioimpedance sensor and the accelerometers, handling data, controlling the display and establishing wireless communication with access points using Bluetooth 5 protocol. Four LEDs were available to notify the user for system power, charging status, battery level and data writing status without activating the display that had a high energy consumption. The button activated the display and could be used for other user interaction.

2) Bioimpedance Sensing: The AD5940 (Analog Devices Inc) analog front end was used to perform bioimpedance sensing. It was digitally configured via SPI to perform four-wire bioimpedance measurements from $1 \mathrm{kHz}$ to 100 $\mathrm{kHz}$. The impedance of the body segment was measured by applying an alternating signal to the body segment and measuring the potential difference between the body segment and the current that flowed through the body segment. The signals were subsequently converted to digital data using a 16 bit ADC. The internal DFT hardware accelerator 


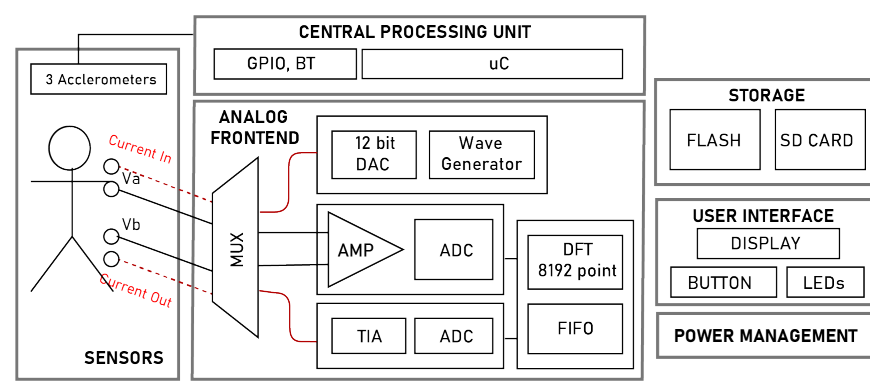

Fig. 1: System level description of the wearable bioimpedance system with main unit featuring the electronics and peripheral sensing units (accelerometers and bioimpedance electrodes).

extracted the real (resistance) and imaginary (reactance) parts of bioimpedance.

3) Accelerometers: Three MMA8451 (NXP Semiconductors) digital accelerometers were used to measure the linear acceleration with respect to positional changes. The accelerometers communicated with the $\mathrm{CPU}$ through the $\mathrm{I}^{2} \mathrm{C}$ interface. The sensor was configured to measure with a $2 \mathrm{~g}$ dynamic range and a resolution of 14 bits.

4) Data Storage: The collected data was stored on either NAND Flash MT29F4G (Micron Technology) or a SD Card.

5) Power Management: A 3.7 V $660 \mathrm{mAh}$ battery (Varta $\mathrm{GmbH}$ ) was used to support the power requirements of the system. The device was either charged through USB or wirelessly through an inductive coil managed by a BQ51013 receiver IC (Texas Instruments). The BQ24073 (Texas Instruments) IC was used to manage the battery charging and power path. Dedicated TPS70933 (Texas Instruments) voltage regulators were used to ensure a $3.3 \mathrm{~V}$ power supply.

6) Wearable: The electronics were integrated into a wearable main unit that can be worn on the forearm (Figure 2). The size of the 3D printed unit was $122 \times 43 \times 14$ $\mathrm{mm}$. The unit provided access to touch proof electrodes and accelerometer connectors, SD card, and USB charging. A custom hand-cuff made of medical foam fixated the hand electrodes and the wearable main unit.

\section{B. Firmware}

The bioimpedance signal was sampled at $20 \mathrm{~Hz}$. Impedance and phase angles were calculated from the raw ADC values. The accelerometer signals were sampled at 30 $\mathrm{Hz}$ and the raw 14 bit signal was converted to $\mathrm{m} / \mathrm{s}^{2}$. The length of the window used to determine the posture was 0.67 $\mathrm{s}$ with a $50 \%$ overlap between consecutive segments.

\section{Methods}

\section{A. Experiments}

To perform functional testing of the device, we conducted a series of measurements on healthy young adults. The aim was to assess the accuracy of the measured signals, the capacity of performing real-time, continuous measurements and to demonstrate the context-awareness of the system. This was achieved with two experiments: a) Performance comparison of the novel device against a commercially

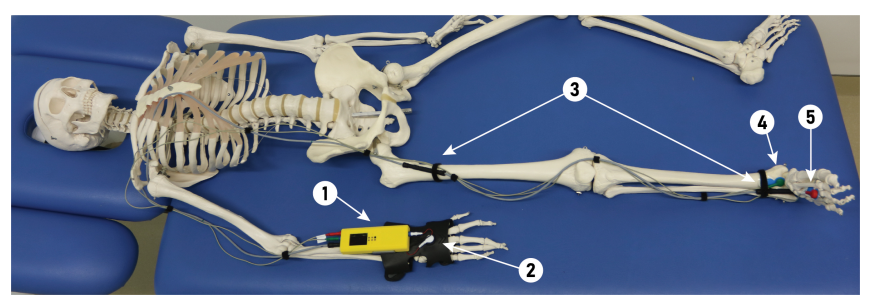

Fig. 2: Wearable, context-aware tetra-polar bioimpedance measurement system. 1) Main unit connecting to 2) wearable cuff the two hand electrodes. 3) upper and lower leg accelerometers, 4) and 5) are the foot electrodes. Upon measurement, this subject showed very high impedance suggesting a high state of dehydration.

available reference device in a stable supine posture, and b) continuous measurement at different postures, including during the transitions between them. The experiments were conducted at ETH Zurich, Balgrist Campus, Zurich, Switzerland. The study was performed according to the Declaration of Helsinki and the protocol was approved by the institutional ethics committee of ETH Zurich (EK2018-N-69).

1) Subjects: After informed and written consent, healthy volunteers were recruited. The inclusion criteria were: older than 18 years, no existing skin disorders or allergies, no metallic or electrical implants, no pregnancy, and provision of informed consent. Prior to clinical measurements, anthropometric data such as BMI, age, ethnicity, height and weight were collected.

2) Placement of Electrodes: Following the collection of anthropometric data, the electrodes and accelerometers were placed on the body. The subject was requested to lie down in a supine position for at least 5 min before starting the measurements in order to stabilize the hydrostatic pressure acting on the column of fluid in the body. The electrodes (EL500, BIOPAC Systems Inc) were placed on the subject after thoroughly cleaning the skin with medical ethanol. The sensing electrodes were placed at the wrist and ankle while the source electrodes were placed centrally on the metacarpals on the palm and metatarsals on the feet (Figure 2). The electrodes were connected to the measurement devices with long shielded medical instrumentation cables matching the respective device connectors. For all subjects, the electrodes were placed on the right limbs. To enable electrode switching between the devices during the experimental protocol, the main unit was not placed directly on the arm, but kept adjacent to the subject.

3) Placement of Accelerometers: Two accelerometers were needed to faithfully reproduce positional orientation [8] and were placed using velcro on the ankle (adjacent to the sensing electrode) and on the anterior quadriceps region of the thigh (Figure 2). The third accelerometer was embedded in the main unit and could not be used for the experiments (see above). Therefore, a replacement accelerometer was placed on the upper limb.

4) Experimental Protocol: We performed two experiments: a) Accuracy and precision testing: During this part of the protocol, the subjects remained in supine position. The measurement cycle started with a measurement with the 
reference device (BIACorpus, Medi-cal Healthcare GmbH, Germany). After switching the electrodes to the wearable device, 6 frequency sweeps of $5 \mathrm{~s}$ were recorded $(30 \mathrm{~s})$. This cycle was repeated 3 times. b) Posture variation and continuous measurement testing: The wearable device was changed to continuously measure bioimpedance and accelerometer data. The subject followed specific positional changes that altered every $40 \mathrm{~s}$. The fixed order of supine-sitting-standingsitting-supine-standing-supine for each subject ensured that every possible transition could be acquired and compared.

\section{B. Post-processing}

The data recorded from the reference and wearable device were transferred to a PC for further analysis using MATLAB (R2019b, Mathworks Inc). The bioimpedance values retrieved from the system were filtered using a median filter to remove spiking artifacts from the signals. The accelerometer signal was filtered using a $2^{\text {nd }}$ order Butterworth lowpass filter with a cutoff frequency at $3 \mathrm{~Hz}$. The posture orientation angles were calculated such as

$$
\theta=\arccos \left(a_{z} / \sqrt{a_{x}^{2}+a_{y}^{2}+a_{z}^{2}}\right)
$$

The data from the upper and lower leg accelerometer pair were used to identify the angles associated with the body postures. Posture classification was performed using a decision tree. Data was divided into a training (8 subjects) and a hold-out test set ( 2 subjects). Ten models were trained using cross-validation with class balancing before training. The model with the least validation error was selected.

\section{Performance evaluation}

Pearson's correlation coefficient $r$ and Bland-Altman plots were generated to compare the agreement between the wearable device and BIACorpus. The bias, limits of agreement (LoA) and their 95\% confidence intervals (CI) were calculated. The LoA were computed after testing for normality of the difference in measurements using the Anderson-Darling test for normality at a 5\% significance level. The posture classification was evaluated on the test set for accuracy, sensitivity, specificity and precision.

\section{RESUlTS}

We measured 9 females (age: $26.6 \pm 3.2$ y, BMI: 22.7 \pm 2.8 ) and 5 males (age: $27.3 \pm 2.4$ y, BMI: $23.8 \pm 1.6$ ), of whom 11 had European, two East Asian, and one South American ethnic background. Four recordings were excluded from the performance comparison, due to faulty data from the reference device (3) or a faulty connection in the wearable device (1). The internal connector failed during motion of the posture changing experiment in 4 recordings which were subsequently excluded from the respective analysis.

\section{A. Performance}

The impedance measurements between the wearable and reference device had high correlation $(r=0.98, \mathrm{p}<0.0001)$. The Bland-Altman agreement plot revealed a bias of $4.5 \pm 13.0 \Omega($ CI $[-6.1,-3.0] \Omega)$ and LoA of $[-30.0,21.0]$ $\Omega$ (CI [-32.6, 23.6] $\Omega$, Figure 3).

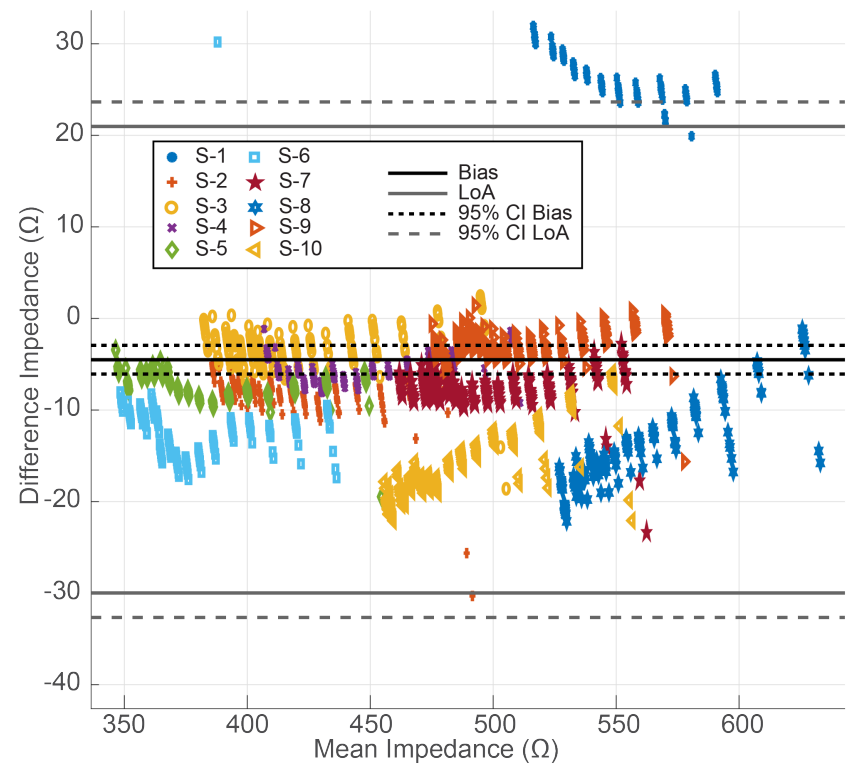

Fig. 3: Impedance agreement between wearable and reference device clustered by subject.

TABLE I: Power consumption during different device states

\begin{tabular}{|l|c|c|c|c|}
\hline \multirow{2}{*}{ Component } & \multicolumn{4}{|c|}{ Current (mA) @ 3.3V } \\
\cline { 2 - 5 } & Sleep & Idle & Meas & Meas \& Disp \\
\hline MMA8451 (x3) & $0.000^{\mathrm{a}}$ & 0.005 & 0.072 & 0.072 \\
AD5940 & $0.000^{\mathrm{a}}$ & 0.009 & 9.100 & 9.100 \\
BLE654 & $0.000^{\mathrm{a}}$ & 0.003 & 0.370 & 0.370 \\
Display & $0.020^{\mathrm{b}}$ & 0.780 & 0.780 & 25.000 \\
\hline Total & 0.020 & 0.797 & 10.31 & 34.542 \\
\hline
\end{tabular}

\begin{tabular}{|c|c|c|c|c|}
\hline & & Reference & & \\
\hline & supine & sitting & standing & precision \\
\hline ذ supine & $678340.7 \%$ & $2281.4 \%$ & $1460.9 \%$ & $94.8 \%$ \\
\hline sitting & $1851.1 \%$ & $445526.7 \%$ & $1360.8 \%$ & $93.3 \%$ \\
\hline standing & $890.5 \%$ & $1170.7 \%$ & $451827.1 \%$ & $95.6 \%$ \\
\hline sensitivity & $96.1 \%$ & $92.8 \%$ & $94.1 \%$ & accuracy \\
\hline specificity & $96.1 \%$ & $97.3 \%$ & $98.3 \%$ & $94.6 \%$ \\
\hline
\end{tabular}

Fig. 4: Confusion matrix showing the position classification performance on the test set $(\mathrm{N}=16657)$.

\section{B. Continuous Bioimpedance}

The power consumption during various system states was low with $0.8 \mathrm{~mA}$ during idle and $10.31 \mathrm{~mA}$ during measurement (Table I). Most power was consumed by the LCD display which can be turned off by the CPU for continuous measurements. As shown in Fig. 5, a continuous spectroscopic bioimpedance signal could be obtained, with a full spectrum sweep performed every $5 \mathrm{~s}$.

\section{Context Awareness}

The test set accuracy on the posture detection was $94.6 \%$ (Fig 4). Fig. 5 illustrates the bioimpedance spectroscopy and the posture changes as classified from the upper and lower leg accelerometers. It was apparent that during sitting the baseline of the bioimpedance was reduced. 


\section{Discussion}

The wearable device developed in this work was a position-aware bioimpedance system. The low power consumption enabled up to $60 \mathrm{~h}$ of continuous measurement operation which could be extended with an optimized power plan. The operation of the wireless data modules will contribute to increased power consumption, but this feature was not yet implemented at time of testing. The system had control over the powering and writing sequence of the storage peripherals, thereby limiting power drain by these components. Considering these features, the device was able to perform recording continuously and in real time.

\section{A. Performance}

The bioimpedance values measured by the wearable device showed high agreement with the values from BIACorpus. As expected, the measured impedance values decreased with an increase in frequency for both devices. Figure 3 indicated an appreciable degree of agreement between the wearable and reference. The negative $95 \%$ confidence intervals for the mean indicated an overall negative bias. S1 was the exception where a strong positive bias of unclear origin was observed. Inter-subject variations did occur. While many subjects showed high agreement, subjects S-1, 6, 8, 10 displayed less agreement. However, the observed intrasubject variation was low. Furthermore, a trend between the difference measured and the mean values was observable. This indicated a relationship between the differences measured and the frequency used for measurements. As the frequency increased, the difference increased for most subjects. This trend could be attributed to a system drift during high frequency measurements. Further investigations and experiments will provide a better understanding of these observations.

\section{B. Context Awareness}

The angles measured using the upper leg accelerometer data was crucial for differentiating supine and sitting position from standing, while the angles measured from the lower accelerometer data was crucial for differentiating between standing and sitting from supine. Therefore, the use of an upper-lower leg pair for posture detection was well justified.

Consistent information regarding motion during bioimpedance measurements is required to compensate posture related signal variability. A previous study indicated the influence of posture on the bioimpedance signal [9], which is an observation that we were able to reproduce.

In this work, we explored context-aware sensing to provide more information regarding posture, time-locked with the acquisition of the bioimpedance signal. The need for wired connections that caused a loss in data has been eliminated in a subsequent design iteration. Therefore, we believe that the proposed context-aware system has the capacity to improve the interpretation of bioimpedance data that was obtained continuously over longer periods of time and making it a suitable approach for clinical applications.

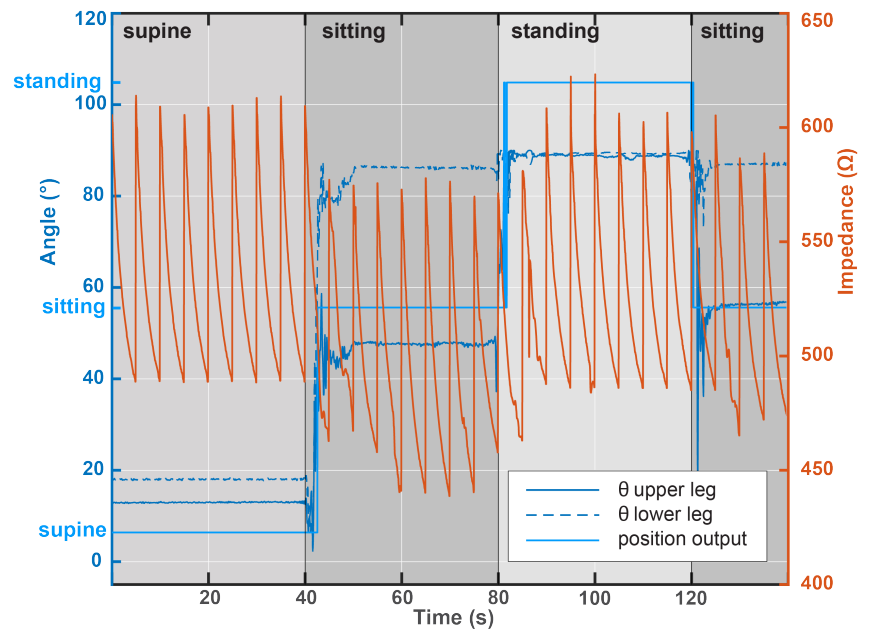

Fig. 5: Bioimpedance spectroscopy signal (red, right axis) and angle data from accelerometers placed at the upper and lower leg (blue, left axis) measured during the changing postures protocol. The position classifier output is shown in light blue.

\section{ACKNOWLEDGMENT}

We thank Prof. C. Spengler and P. Eichenberger from the Exercise Physiology Lab for facilitating access to the reference bioimpedance device, L. Schnorf and J. Conrad for conceptualizing the wearable design for the device, $\mathrm{K}$. Dheman and S. Hofstede for drafting the ethics proposal and experimenting with earlier prototypes, all the volunteers for participating in the study, the team at Oxford University Clinical Research Unit (OUCRU) in Vietnam for the many fruitful discussions, and Marcello, our photo model.

\section{REFERENCES}

[1] J. Ferreira, F. Seoane, and K. Lindecrantz, "Portable bioimpedance monitor evaluation for continuous impedance measurements. towards wearable plethysmography applications," in 2013 35th Annual International Conference of the IEEE Engineering in Medicine and Biology Society $(E M B C)$. IEEE, 2013, pp. 559-562.

[2] P. Wabel et al., "Importance of whole-body bioimpedance spectroscopy for the management of fluid balance," in Blood Purification, vol. 27, no. 1, 2009, pp. 75-80.

[3] F. Ibrahim et al., "A novel approach to classify risk in dengue hemorrhagic fever (DHF) using bioelectrical impedance analysis (BIA)," IEEE Transactions on Instrumentation and Measurement, vol. 54, no. 1, pp. 237-244, jan 2005.

[4] H. C. Lukaski, "Evolution of bioimpedance: A circuitous journey from estimation of physiological function to assessment of body composition and a return to clinical research," European Journal of Clinical Nutrition, vol. 67, pp. S2-S9, 2013.

[5] E. C. Rush et al., "Validity of hand-to-foot measurement of bioimpedance: Standing compared with lying position," Obesity, vol. 14, no. 2, pp. 252-257, 2006.

[6] D. Buxi et al., "Correlation between electrode-tissue impedance and motion artifact in biopotential recordings," IEEE Sensors Journal, vol. 12, no. 12, pp. 3373-3383, 2012.

[7] D. H. Libraty et al., "Assessment of body fluid compartment volumes by multifrequency bioelectrical impedance spectroscopy in children with dengue," Transactions of the Royal Society of Tropical Medicine and Hygiene, vol. 96, no. 3, pp. 295-299, 2002.

[8] S. Hersek et al., "Wearable vector electrical bioimpedance system to assess knee joint health," IEEE Transactions on Biomedical Engineering, vol. 64, no. 10, pp. 2353-2360, 2016.

[9] G. Medrano, S. Leonhardt, and P. Zhang, "Modeling the influence of body position in bioimpedance measurements," in 2007 29th Annual International Conference of the IEEE Engineering in Medicine and Biology Society (EMBC). IEEE, 2007, pp. 3934-3937. 